\title{
Non-axisymmetric complete flow conditioning gauzes
}

\author{
Alejandro Castillo Pardo ${ }^{1}$ (D . James V. Taylor ${ }^{1}$ (I)
}

Received: 3 May 2021 / Revised: 23 July 2021 / Accepted: 22 August 2021 / Published online: 14 September 2021

(c) The Author(s) 2021

\begin{abstract}
This paper presents a novel methodology for the design of a gauze that produces distributions of stagnation pressure, swirl angle, pitch angle and turbulence intensity, tailored in both the radial and circumferential directions. A distortion gauze is made from a large number of small-scale circumferential and radial blades with tailored thickness and camber distributions. By controlling the blade design independently in both the radial and circumferential directions, the target inflow pattern can be achieved. 1D correlations are used to initialise the blades and they are refined using full 3D CFD simulations. The final design is additively manufactured for use in rotating rigs. In this paper, the method has been used to reproduce four target inflow patterns with large variations in stagnation pressure and flow angularity. Two examples model the inlet flow distortion seen at the aerodynamic interface plane of an aft-mounted boundary layer ingesting fan. The final two examples model the inlet distortion at inlet to an axial compressor spool caused by upstream structural struts in a swan neck duct. The gauzes are shown to replicate the structures of the target flow in an experimental test. These kind of flow structures would be extremely difficult or impossible to replicate in an experiment in any other way.
\end{abstract}

\section{Graphical abstract}

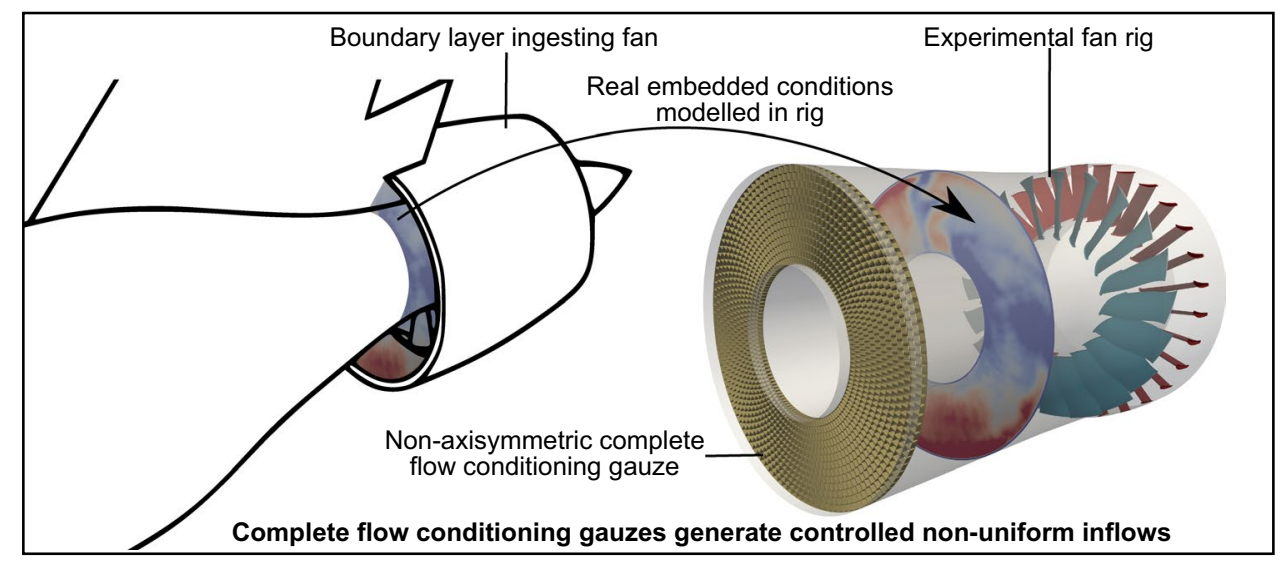

\section{Abbreviations}

a, b Roach's correlation constants

h Stage span

r Radial coordinate

V Flow velocity

$\alpha \quad$ Swirl flow angle

$\beta \quad$ Gauze porosity

Alejandro Castillo Pardo ac2181@cam.ac.uk

1 Whittle Laboratory, Department of Engineering, University of Cambridge, Cambridge, UK
$\Delta C_{p_{0}} \quad$ Stagnation pressure drop coefficient

$\zeta \quad$ Pitch flow angle

$\theta \quad$ Circumferential coordinate

$\lambda \quad$ Radial blade angle

$\chi \quad$ Circumferential blade angle

$\Omega \quad$ Blade rotational speed

(-) Average value

()$_{\text {mix }} \quad$ Value at the target plane

() $)_{\mathrm{TE}} \quad$ Value at the trailing edge

() $\quad$ Axial component

()$_{r} \quad$ Circumferential component 
() $\quad$ Radial component

BLI Boundary layer ingestion

CFD Computational fluid dynamics

RANS Reynolds-Averaged Navier-Stokes

\section{Introduction}

With the advent of tighter engine-intake-airframe integration to reduce emissions, increasing levels of distortion are expected in all turbomachinery components. This distortion takes the form of large swings in flow angle, both swirl and pitch, along with strong stagnation pressure gradients. To understand trades in performance with tighter integration, a new experimental method is required that can model the real embedded flow structures in a laboratory environment. It is possible to tailor the inflow pattern to achieve any flow condition that varies in both radial and circumferential directions using additively manufactured complete flow conditioning gauzes. Only with this approach it is possible to decouple and quantify the effect of the increased distortion on the individual turbomachinery components. In addition, this method allows increased certainty and reproducibility in the comparison between experiments and simulation: it is now possible to run the same experiment with different inlet boundary conditions, just as an engineer would use multiple CFD simulations to probe sensitivities.

This paper presents the methodology for the design of non-axisymmetric complete flow conditioning gauzes along with four examples of applications in both boundary layer ingesting fans and compact core compressors. The method to design a gauze with variable porosity and blades with variable turning builds upon the paper by Taylor (2019) for axisymmetric flow conditioning gauzes. Figure 1 shows how the geometry of the gauze can be varied in both radial and circumferential directions to achieve a target inflow that models that seen at the inlet to an aft-mounted fan that is ingesting the boundary layer from an aircraft's fuselage. The gauze is 3D printed and mounted in a rotating rig in a laboratory so that the effect of this inlet condition on the experiment can be quantified and understood.

In the field of fan-distortion two different methodologies have been identified from the literature. The first methodology is the integration of the fan stage into a wind tunnel that is purpose built to replicate some or all the upstream geometry of the real aircraft. A Boundary Layer Ingesting (BLI) fan semi-buried in the floor of a wind tunnel is presented by Arend et al. (2017), whilst a wind tunnel facility built for the analysis of fan-intake interaction under crosswind conditions is reported by Harjes et al. (2020).

The second methodology consists of the use of distortion generation devices at the inlet of the fan rig to replicate the inflow of interest at the fan face. Tests carried out with

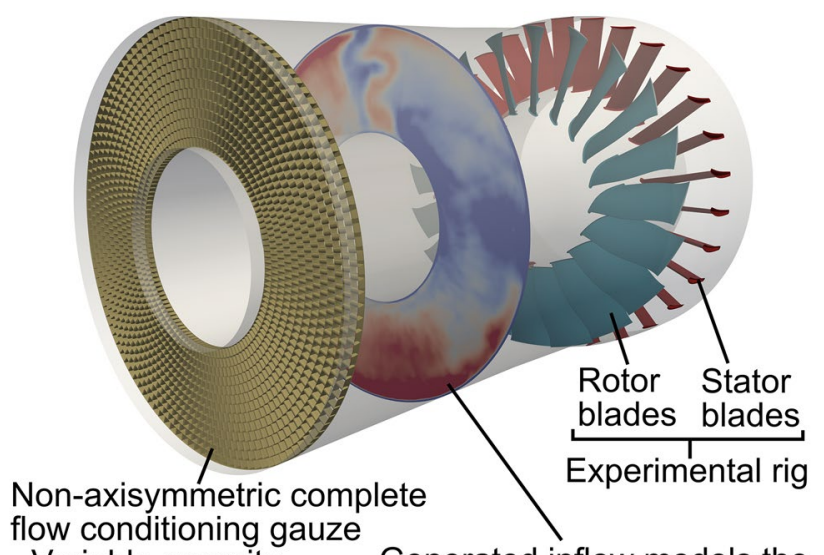

- Variable porosity

- Variable turning

Generated inflow models the real embedded conditions

Fig. 1 Non-axisymmetric complete flow conditioning gauze

fan rigs equipped with distortion generators are presented by Gunn and Hall (2014) for a semi-buried BLI fan and by Castillo Pardo and Hall (2021) for a $360^{\circ}$ fuselage BLI fan. The design of this second type of high-fidelity distortion generation devices remains a challenge, but it offers numerous advantages. Firstly, it can be more flexible, as different magnitudes or types of distortion can be investigated with the same fan rig by changing only the distortion generating device. Secondly, the overall cost, complexity, size, and duration of the experimental tests can all be reduced. In a wind tunnel embedded fan, a bypass flow is required, and this can become unwieldy if compromises on the size of the fan rig itself are not made.

Traditionally, stagnation pressure distortion is generated by a combination of distortion screens, grids or endwall thickeners (Place et al. 1996; Lucas, et al. 2014), whilst swirl is generated with inlet guide vanes (Wheeler et al. 2006; Houghton and Day 2011). Gunn and Hall (2014) designed and $3 \mathrm{D}$ printed a precisely controlled variable porosity gauze to replicate the axial velocity profile found at the intake of a semi-buried BLI fan. The fine hole resolution resulted in a relatively smooth flow distribution. However, secondary flows were not considered. In contrast, the device presented by Guimarães et al. (2018) comprises discrete turning blades that replicate the vortical structures found in military installations. The reduced blade count in this device results in higher loading. Consequently, the stronger blade wakes are still seen at the fan face. This device was combined with distortion screens by Frohnapfel et al. (2020) to add stagnation pressure distortion to the attained vortical flow. Taylor (2019) presented a flow conditioning gauze that controls simultaneously the radial distributions of swirl and stagnation pressure. The gauze, which was additively manufactured, was successfully applied to replicate a multi-stage embedded profile in compressors. However, the method was 
restricted to axisymmetric inflows with zero pitch angle. In this paper, the method is developed so that it can be used to reproduce any target inflow.

\section{Gauze design}

This section presents the methodology for the design of a non-axisymmetric complete flow conditioning gauze. Figure 2 shows a flowchart of the entire process and a reference to the equations that are used at each stage. This is broken down into six parts that are described in this section. The process starts by scaling a target flowfield to the operating conditions of the experimental rig, then 1D correlations are used to initialise the gauze porosity and exit blade angles. Next, 3D CFD is used iteratively to refine the gauze geometry: three parts include details of the blading, meshing and simulation and the streamline tracking and feedback process used to update the geometry. In the last part, the manufacturing method and its considerations are presented.

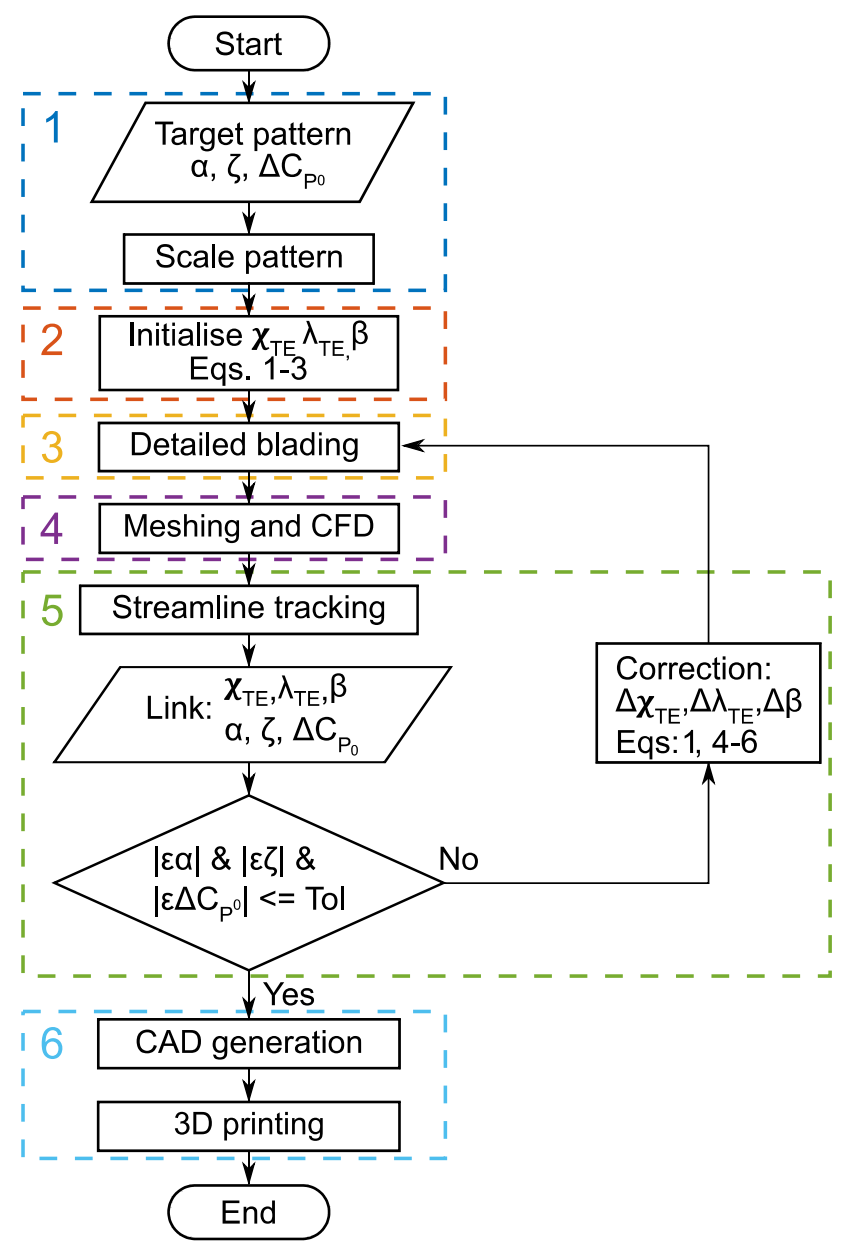

Fig. 2 Gauze design flowchart

\subsection{Target distortion scaling}

A flow conditioning gauze aims to generate a specific flowfield upstream of an experimental turbomachinery rotating rig. This flowfield can be obtained from different sources. Examples include numerical predictions or experimental measurements at the intake of a fan or at the outlet of the preceding compressor blade row. The rotational speed of the experimental rig $(\Omega)$ might differ from the speed of the system where the flowfield was obtained. Therefore, the flowfield needs to be scaled.

To preserve the velocity triangles seen in a rotating rig experiment, the local flow coefficient $\left(\mathrm{V}_{\mathrm{x}} / \mathrm{U}\right)$ must be kept constant. To maintain the swirl $(\alpha)$ and pitch $(\zeta)$ angles of the inflow, defined in Fig. 3, the ratio between the velocity components must remain unchanged. Considering both constraints, a new velocity flowfield scaled to the speed of the rig of interest is generated. The stagnation pressure field associated with the scaled velocity now constitutes the target distribution to be attained by the gauze design. The turning of the gauze must also match the target swirl and pitch angles.

\subsection{Gauze initialisation}

The second step initialises the distributions of porosity and blade angles across the gauze. It requires the knowledge of the target flowfield, calculated in the previous step, and the velocity field upstream of the gauze. The latter can be either measured or estimated and enables the calculation of the dynamic head approaching each passage. The drop in stagnation pressure required to achieve the target profile is normalised by the inlet dynamic head to generate the stagnation pressure drop coefficient $\left(\Delta C_{p_{0}}=\left(p_{0, u p}-p_{0}\right) /\left(p_{0, u p}-p_{u p}\right)\right)$, where $p_{0, u p}$ and $p_{u p}$ are the stagnation and static pressure upstream of the gauze, respectively. Roach (1987) proposed a correlation between the porosity of a grid $(\beta)$ and its stagnation pressure drop coefficient $\left(\Delta C_{p_{0}}\right)$. The correlation, presented in Eq. 1 in the same form as Taylor (2019), is used to estimate the initial distribution of porosity.

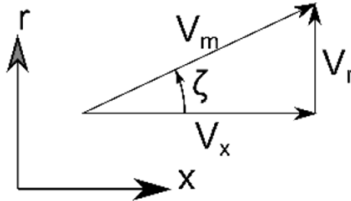

Pitch angle: $\zeta$

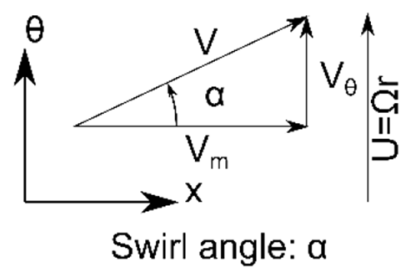

Fig. 3 Flow angles definition 
$\boldsymbol{\beta}=\left(\frac{1}{\left(\frac{\Delta C_{p_{0}}}{a}\right)^{\frac{1}{b}}+1}\right)^{\frac{1}{2}}$

Roach's correlation uses two constants: $a$ and $b$. These are a function of the Reynolds number, Mach number and grid geometry. Values of $a=0.52$ and $b=1.0$ have been used in this study as per Taylor (2019). The porosity is defined as the ratio of the open area to the total passage area at the trailing edge.

By varying the mean value of porosity, any number of gauzes can be designed to produce the desired stagnation pressure drop coefficient pattern; these will differ by their mean loss. The choice of mean porosity affects other aspects of the design: turbulence intensity, manufacturability, and operability. A reduced mean porosity causes a greater mean stagnation pressure loss, possibly increasing the power demand on auxiliary exhaust suction systems. Additionally, it leads to thicker trailing edges, which ultimately increase the mean turbulence intensity upstream of the rotating stage. Moreover, thicker trailing edges improve the structural integrity of the gauze. This is beneficial if an additive manufacturing process is used, however it can be detrimental if a machining process is used-the reduced open passage area reduces the accessibility for cutting tools. All gauzes shown in this paper were 3D-printed in UV-cured resin. The mean porosity was set as high as possible whilst ensuring a minimum trailing edge thickness of $0.35 \mathrm{~mm}$ for the low-speed fan rig and $0.5 \mathrm{~mm}$ for the medium-speed compressor rig.

Next, the distributions of circumferential $\left(\chi_{T E}\right)$ and radial $\left(\lambda_{T E}\right)$ blade trailing edge angles are initialised. At this stage the flow is assumed to remain attached until the blade trailing edge and the deviation is negligible, this leads to the approximation of blade angles equal to flow angles at the trailing edge $\left(\chi_{T E}=\alpha_{T E}\right.$ and $\left.\lambda_{T E}=\zeta_{T E}\right)$. The effect of mixing downstream of the gauze is included as it acts to increase the flow angles. The increase is related to the local porosity with Eq. 2 as in Taylor (2019) and Eq. 3 which follows.

$$
\begin{aligned}
& \tan \left(\chi_{T E}\right)=\boldsymbol{\beta} \cdot \tan \left(\boldsymbol{\alpha}_{\text {mix }}\right) \\
& \tan \left(\lambda_{T E}\right)=\boldsymbol{\beta} \cdot \tan \left(\zeta_{\text {mix }}\right)
\end{aligned}
$$

\subsection{Blading}

The gauzes are comprised of thousands of small-scale passages bounded by radial and circumferential blades. Radial blades turn the flow in the circumferential direction, generating swirl. In contrast, circumferential blades turn the flow radially, producing a pitch component. The chosen number of passages in the radial and circumferential direction determines the smoothness of the flowfield, the structural integrity and the manufacturability. A high number of blades is desired to reduce the individual blade loading, reducing the strength of the wake and improving the smoothness of the flowfield. However, a greater number of passages will result in thinner trailing edges and compromised structural integrity during the manufacturing process and rig operation. The choice of radial blade count also determines the pitch to height ratio of the passage, which should be kept close to unity to minimise secondary flows. The choice of circumferential passage count is more complex for low hubto-tip ratio rigs, where the passages near the hub perform optimally with a low number whilst the passages near the casing operate better with a higher count. For the BLI fan application, 13 radial and 120 circumferential passages were chosen as a compromise between hub and casing aerodynamic performance, smoothness and structural integrity. For the compressor application, a combination of 9 radial and 360 circumferential passages was chosen.

All blades are cambered to be able to produce turning. The thickness and camber vary along a blade and they are determined by the porosity, trailing edge thickness and blade angle of the surrounding passages. Circular arc camber lines with maximum thickness location at $25 \%$ of chord were generated to reduce flow diffusion and separation across the passage. Alternating rows of blades are offset in the circumferential direction by half of a passage pitch. This increases grid rigidity and improves downstream mixing, and circumferential blades are also crenelated to increase mixing. Radial blades are stacked on a parabolic lean profile to prevent corner separations in passages that have high turning. The magnitude of the lean is scaled across the whole gauze design depending on the magnitude and direction of the required turning. A render of the blading in one of the compact compressor designs is shown in Fig. 4, Further details on the individual modelling of each blade can be found in Taylor (2019).

\subsection{Meshing and simulation}

To refine the design of the gauze geometry, a 3D CFD method is used to predict the flow within the passages of the gauze as well as the mixing process before it reaches the target plane at inlet to the experiment. The structured multiblock RANS solver TURBOSTREAM was selected and is introduced and validated in Brandvik and Pullan (2010). The entire gauze is meshed and solved along with the downstream rotating fan or compressor stage coupled with a mixing plane and the one equation Spalart-Allmaras turbulence model. In the case of the BLI fan, the gauze requires a full annulus calculation. In the case of the compact compressor, 


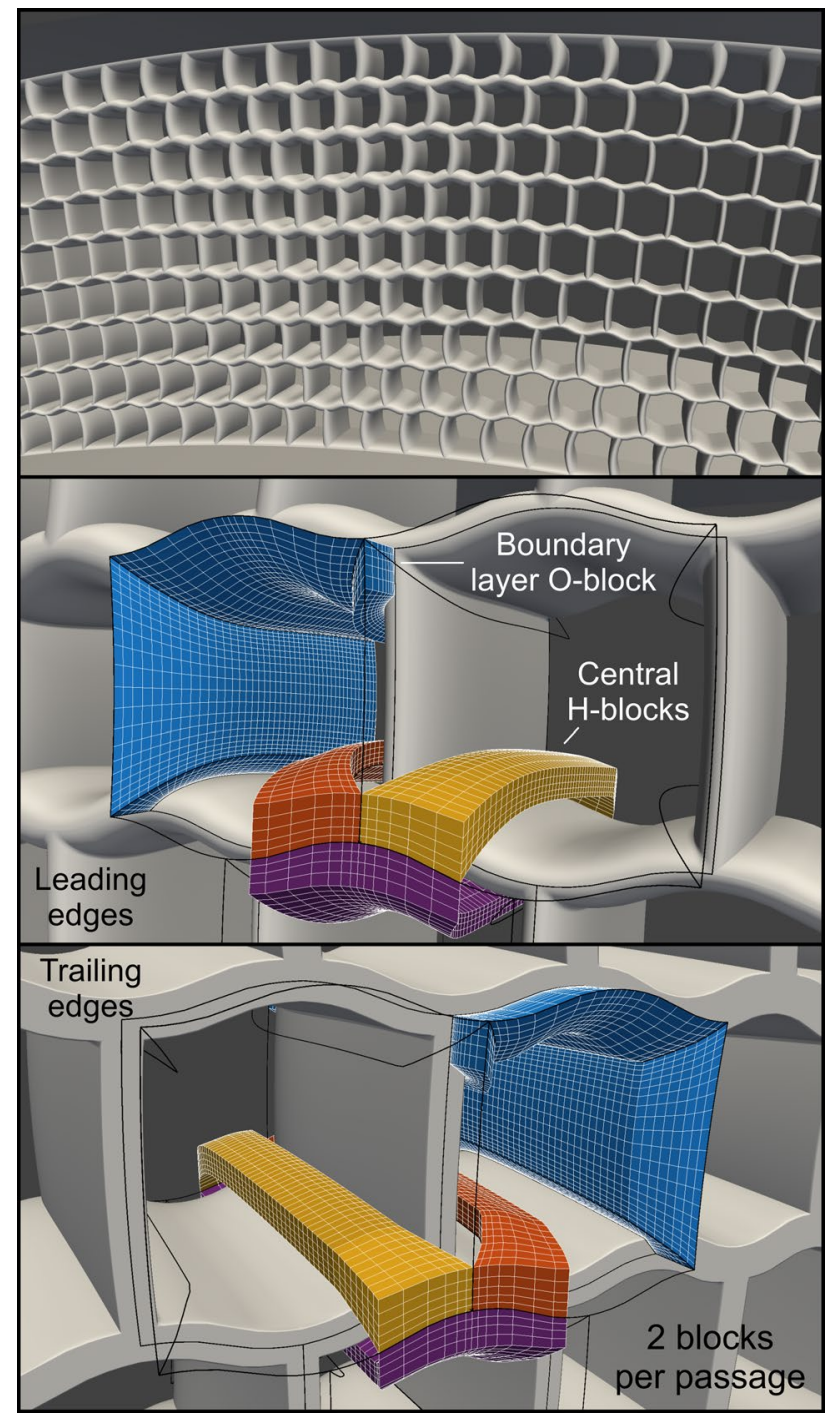

Fig. 4 Structured multi-block mesh strategy

the gauze models the flow downstream of 12 structural struts and so only $1 / 12$ th of the annulus is simulated.

The blocking is refined compared to that used in Taylor (2019), Fig. 4 shows different elevations of the improved strategy. Two blocks are used per passage: the O-block and the H-block. The subset of the mesh shown in blue shows the boundary layer O-block, which wraps around all 4 of the internal surfaces of the passage as well as the leading and trailing edges. Cells can be clustered on the walls to give $\mathrm{a} \mathrm{y}^{+}<1$ with minimal skewness. The subsets of the mesh shown in red, yellow and purple show the central H-blocks from three different passages. The figure shows how these H-blocks all meet at the corners between the passages. The faces from all H-blocks are then patched to a single inlet block upstream and a single outlet block downstream.

The meshing is performed automatically in MATLAB as part of the iterative design process, with 70,000 cells used per gauze passage. Fan and compressor blades were meshed using an $\mathrm{O} 4 \mathrm{H}$ topology with $\mathrm{y}^{+}<1$, a total of 4.4 and 1.2 million cells were used per blade passage for the fan and compressor stage, respectively. Simulations of the flow are run on NVIDIA K20 GPUs as part of the University of Cambridge CSD3 cluster. The full annulus BLI fan case takes $4.5 \mathrm{~h}$ to run on 32 GPUs, while the $1 / 12^{\text {th }}$ annulus compact compressor case takes $4 \mathrm{~h}$ to run on 4 GPUs.

\subsection{Feedback loop}

The numerical results of the 3D CFD model were used as part of a closed feedback loop to minimise the discrepancy between the target and the predicted flowfields. Due to the angularity and asymmetry of the flows of interest, both radial and circumferential displacements take place downstream of the gauze. For a given streamline, the radial $(\Delta r)$ and circumferential displacements $(\Delta \theta)$ are defined as the change in radial and circumferential coordinates between the gauze trailing edge and the target plane. A streamline tracking method was used to calculate this displacement and track the flow backwards from the target plane to the individual passages of the gauze that it passes through. In this way, it is possible to ensure the discrepancies between the stagnation pressure, swirl and pitch angle of the target flow pattern result in updates to the porosity and blade angles in the correct part of the gauze. Figure 5 shows an example selection of the streamlines from the compact compressor case, it can be seen that the pitch and yaw angles in the flow are responsible for large scale displacements between the gauze trailing edge and target plane, in some places in order
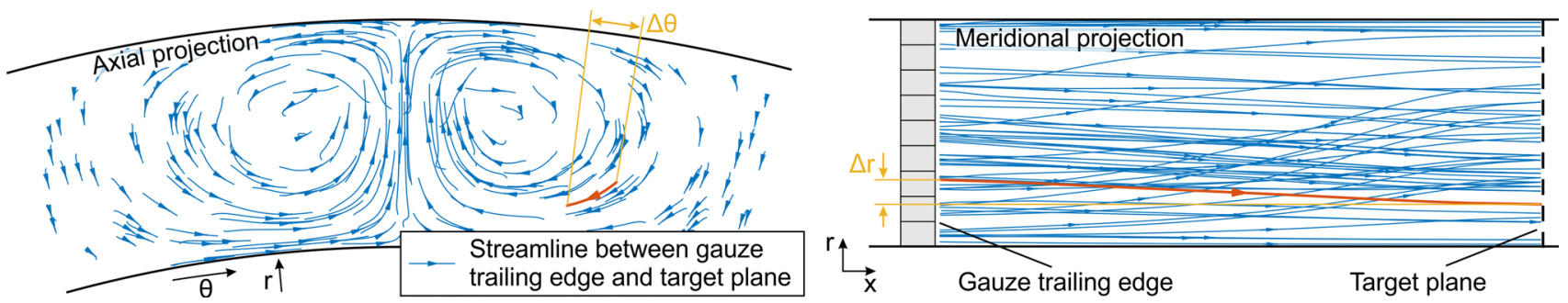

Fig. 5 Selection of tracked streamlines showing displacement downstream of the gauze 
of $40 \%$ of the span. In some gauze applications, the displacements can be small and neglected; in these situations it is easier to use experimental data within the feedback loop.

Once the relative locations between the gauze passages and the target plane have been calculated it is possible to update the design. The blade angles are corrected in a 1:1 ratio with the flow angle discrepancy, as shown in Eqs. 4 and 5. The deviation angle, defined as the difference between the trailing edge blade angle and the flow angle, increases with turning. Consequently, an increase in blade turning leads to a smaller rise in flow angle, stabilising the scheme. A corrected stagnation pressure drop coefficient is calculated using Eq. 6. Two terms are identified in this equation: the first term represents the ideal loss coefficient caused by the local gauze porosity; the second term accounts for the discrepancy in loss between the target and the current numerical estimation. The corrected stagnation pressure drop coefficient is inserted in Eq. 1 to obtain the correction in porosity. The updated porosity is limited to ensure the trailing edge thickness is above the structural minimum. Moreover, a lower limit in the porosity is placed to ensure a minimum open area; a value of $50 \%$ is selected in the examples from this paper. The updated design is smoothed in the radial and circumferential directions before the next iteration to avoid steep discontinuities in geometry.

$$
\begin{aligned}
& \chi_{\mathrm{TE}, \mathbf{n}+1}=\chi_{\mathrm{TE}, \mathbf{n}}+\alpha_{\mathrm{target}}-\alpha_{\mathbf{n}} \\
& \lambda_{\mathrm{TE}, \mathbf{n}+1}=\lambda_{\mathrm{TE}, \mathbf{n}}+\zeta_{\text {target }}-\zeta_{\mathbf{n}} \\
& \Delta C_{p_{0}, n+1}=\boldsymbol{a} \cdot\left(\frac{1}{\beta_{n}^{2}}-1\right)^{b}+\left(\Delta C_{p_{0}, \text { target }}-\Delta C_{p_{0}, \boldsymbol{n}}\right)
\end{aligned}
$$

\subsection{Manufacture}

Flow conditioning gauzes can be manufactured using two processes: additive manufacturing and $\mathrm{CNC}$ machining. Polyjet stereolithography was proved in (Taylor 2019) to provide a good balance between cost, rigidity, and accuracy. The same process has been used for all gauzes designed in this paper. The printer used has an accuracy of $40 \mu \mathrm{m}$ in the radial and tangential direction. The gauzes were printed in the axial direction with a layer thickness of $30 \mu \mathrm{m}$. Flanges were added to the hub and casing to improve structural integrity and allow the gauze to be clamped and installed in the rig, as shown in Fig. 6. CNC machining is an alternative to $3 \mathrm{D}$ printing, however the passage count needs to be reduced to improve tool access, which can reduce the resolution and resultant smoothness of the target flowfield. Machined gauzes are more suitable for high speed applications. Alternatively, direct metal laser sintering can be used

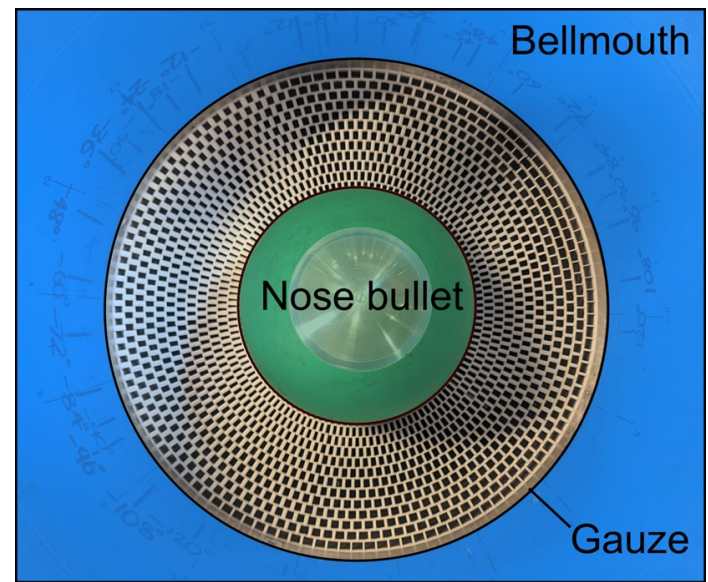

Fig. 6 Photocured resin gauze installed in the BLI fan rig

for high speed application. However, its cost and surface roughness finish make it unsuitable as an engineering tool.

\section{Experimental methods}

Two experimental rotating rigs located at the Whittle Laboratory have been used to demonstrate the example gauze designs in this paper: the BLI fan rig and the Gibbons compressor rig.

The BLI fan rig is a low-speed single-stage fan rig. It was purpose built for the analysis of aft-section boundary layer ingesting (BLI) fan aerodynamics. The rig has a long intake duct which enables the free interaction of the distorted inflow with the rotor blades. A five-hole pneumatic probe mounted on an area traverse system was used to resolve the time-average flowfield at the target plane. This plane is located 30 gauze chord-lengths downstream of the gauze and 40 gauze chord-lengths upstream of the fan. The diameter of the head probe was $1.4 \%$ of the span. The uncertainties of probe measurements were of the order of $\pm 0.5 \%$ and $\pm 1.0 \%$ of the dynamic head for the total and static pressure fields; and of the order of $\pm 0.5^{\circ}$ for flow angles. Due to the non-axisymmetric nature of the flow, full annulus area traverses were required. The traverse system operated in a $36^{\circ}$ sector with 29 endwall clustered radial positions and 37 equally spaced circumferential positions. Rotating the distortion gauze ten times relative to this sector enabled the measurement of the full annulus flowfield. Full details of this rig can be found in Castillo Pardo and Hall (2021) and Allen et al. (2021).

The Gibbons compressor rig is a medium-speed single-stage compressor rig. It has been used for compressor design (Taylor and Miller 2016; Bruni et al. 2020) and measurements of in-service effects such as blade damage (Taylor et al. 2020). In this rig, the target plane is 9 gauze 
chord-lengths downstream of the gauze, and the rotor leading edge is a further 5 chords further downstream. Fivehole probe measurements were made with an area traverse system with the same uncertainties as the BLI fan rig. As the gauzes presented in this paper have a periodicity of 12 and are also symmetric, it was possible to traverse a 1/24th sector of the annulus. The flow was resolved with a grid of 27 endwall clustered radial positions and 61 equally spaced circumferential positions.

\section{Gauze applications}

Two applications are presented in this paper: a boundary layer ingesting fan and a compact core compressor. In both applications, gauzes are designed to replicate the flow at the target plane in the real engine configuration at the target plane of the laboratory rig. This is shown schematically in Fig. 7. Two cases are designed for within each application

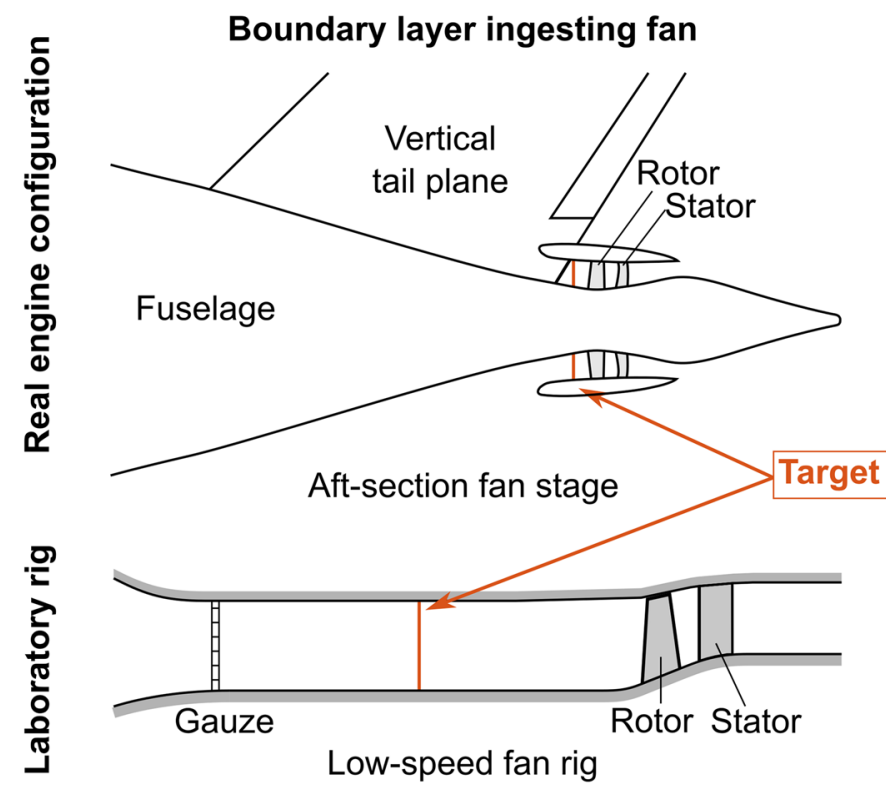

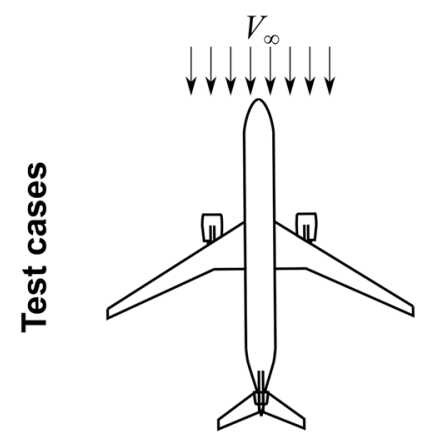

Case 1: cruise

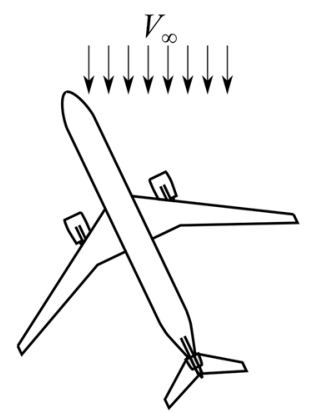

Case 2: $4^{\circ}$ sideslip

to show as examples of different flow features that can be replicated from real engine configurations to be studied and compared within the laboratory rigs.

\subsection{Boundary layer ingesting fan}

The proposed gauze technology has been applied to replicate the flow distortion at the inlet of a BLI fan. The fan of interest is located at the aft-section of CENTRELINE's propulsive fuselage concept (Seitz et al 2021). This aircraft configuration includes a cylindrical fuselage, wings, belly fairing and a vertical tail place upstream of the fan (Fig. 7). The presence of these elements generates a highly distorted inflow with large gradients in the radial and circumferential directions. Three-dimensional numerical simulations of the aircraft including an actuator disc for the aft-section fan were performed by van Sluis (2020). The flow properties found at the aerodynamic interface plane of the aft-section propulsor for two different flight conditions, shown in Fig. 7,

\section{Compact core compressor}
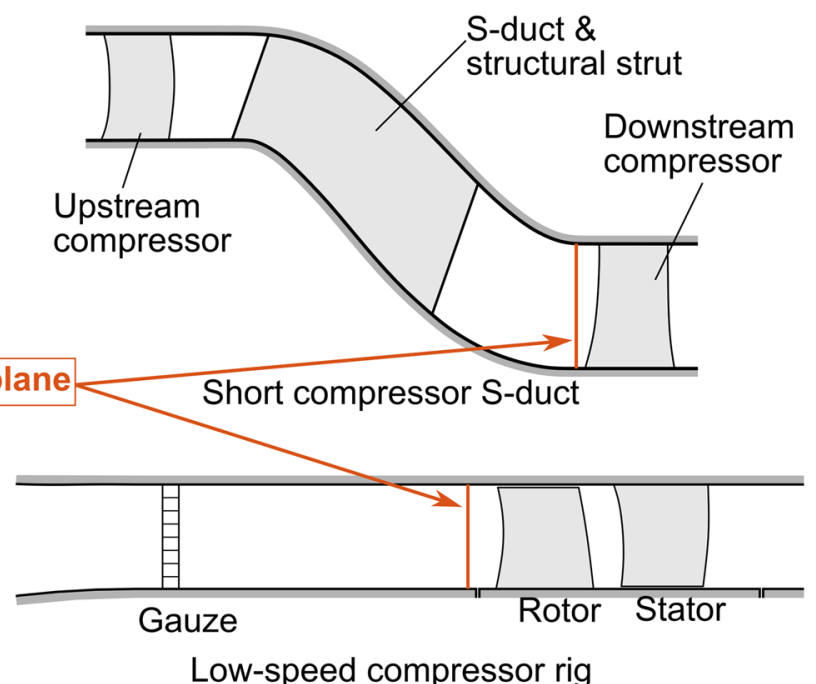

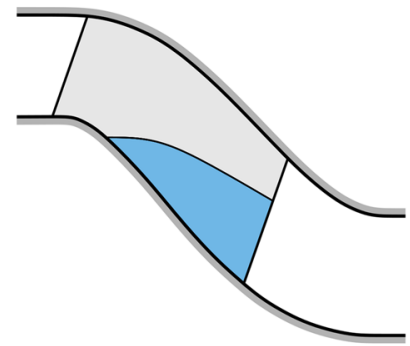

Case 3: Datum duct

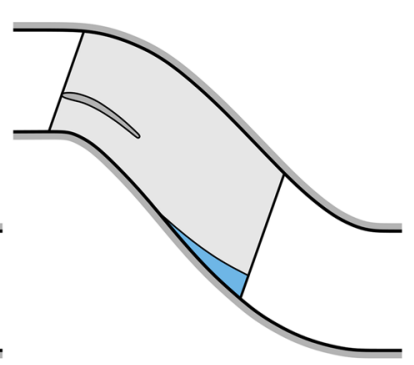

Case 4: Splittered duct

Fig. 7 Sketch of gauze applications 
have been scaled to determine the target flowfields to be generated by the gauzes. For this specific application, the swirl distortion is an order of magnitude higher than the pitch component. Consequently, no attempt to generate pitch distortion has been made.

At the cruise aerodynamic design point, the fuselage is aligned with the freestream. Due to the symmetry of the aircraft about the vertical axis, the inflow at the AIP is symmetric about the same axis. This is confirmed in the target inflow shown in Fig. 8 and Fig. 9 for stagnation pressure loss coefficient and swirl angle, respectively. Note that swirl angle is defined positive in the positive direction of $\theta$. A very localised deficit in stagnation pressure linked to the wake of the vertical tail plane is observed at $\theta=0^{\circ}$. The swirl component of the flow observed between $\theta=-30^{\circ}$ and $\theta=30^{\circ}$ drives higher momentum flow into the wake, filling the wake. Consequently, this local increase in loss is not expected to be replicable. Two larger regions of momentum deficit are found around $\theta \approx \pm 45^{\circ}$. These are associated with horseshoe vortices initiated at the junction between the fuselage and the leading edge of the vertical tail plane. The vortical structures lead to opposing swirl components near the hub and casing. The region of low loss at the bottom of the annulus is linked to two vortices released from the belly fairing. These vortices drive high momentum flow, from the outside of the fuselage boundary layer, into the propulsor. The large top-to-bottom gradient in stagnation pressure induces further upwards flow migration. This leads to a further increase in swirl angle around $\theta \approx \pm 90^{\circ}$. Symmetry has not been enforced during the design process, proving that the solver is able to reach the symmetric solution by itself.

Figure 8 presents the comparison between the target design, numerical predictions and measured stagnation pressure coefficient at the target plane. The loss coefficient is centred around the mean value to highlight the shape of the distortion. The methodology can successfully replicate the main flow features of the stagnation pressure distortion. As previously explained, the localised momentum deficit behind the vertical tail plane has not been attained. The disagreement around the horseshoe vortices suggests the CFD method underpredicts the loss, driving the design solver to lower porosity. Ultimately, the reduced porosity
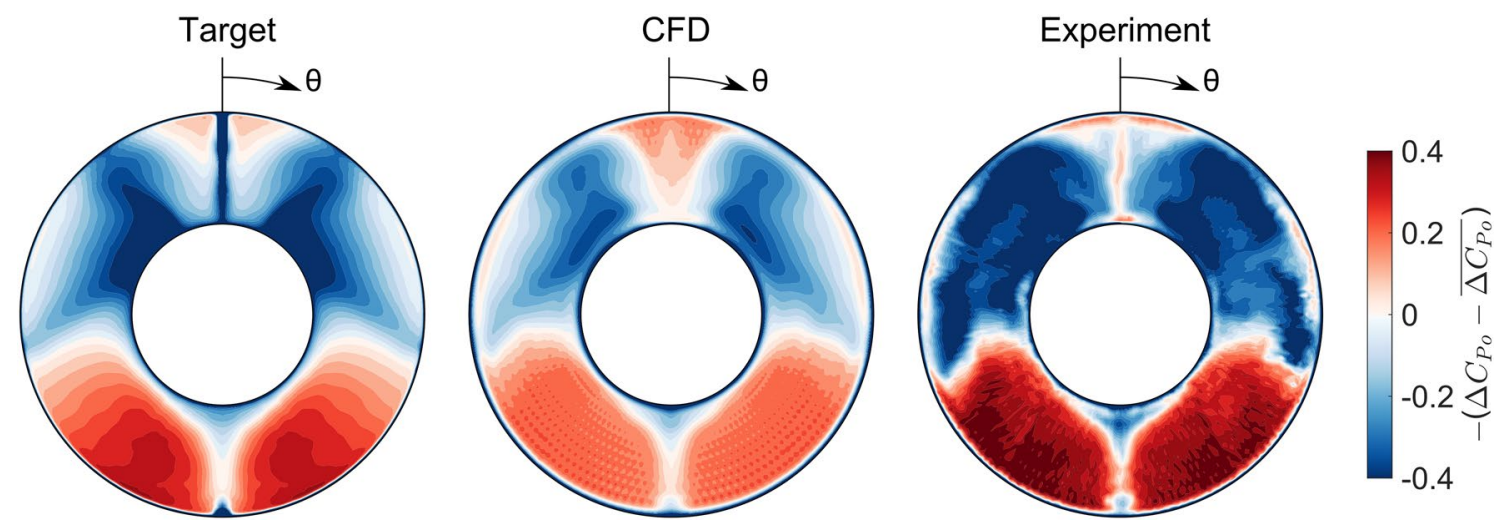

Fig. 8 CFD design and experimental measurements of stagnation pressure coefficient of BLI fan at cruise
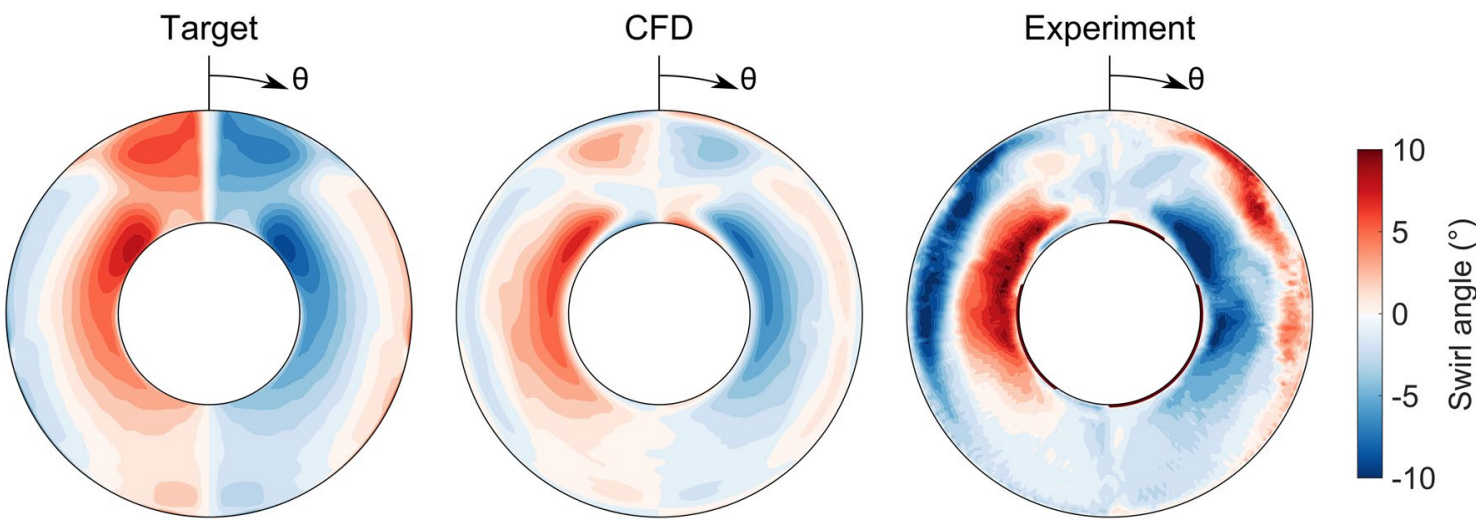

Fig. 9 CFD Design and experimental measurements of swirl angle of BLI fan at cruise 
results in an excessive stagnation pressure drop. Despite the local discrepancies, the main structures of the flow have been reproduced in both the gauze computations and experiments. The swirl angle comparison is presented in Fig. 9. A discrepancy between the target and the experimental results is observed in the regions where loss was numerically

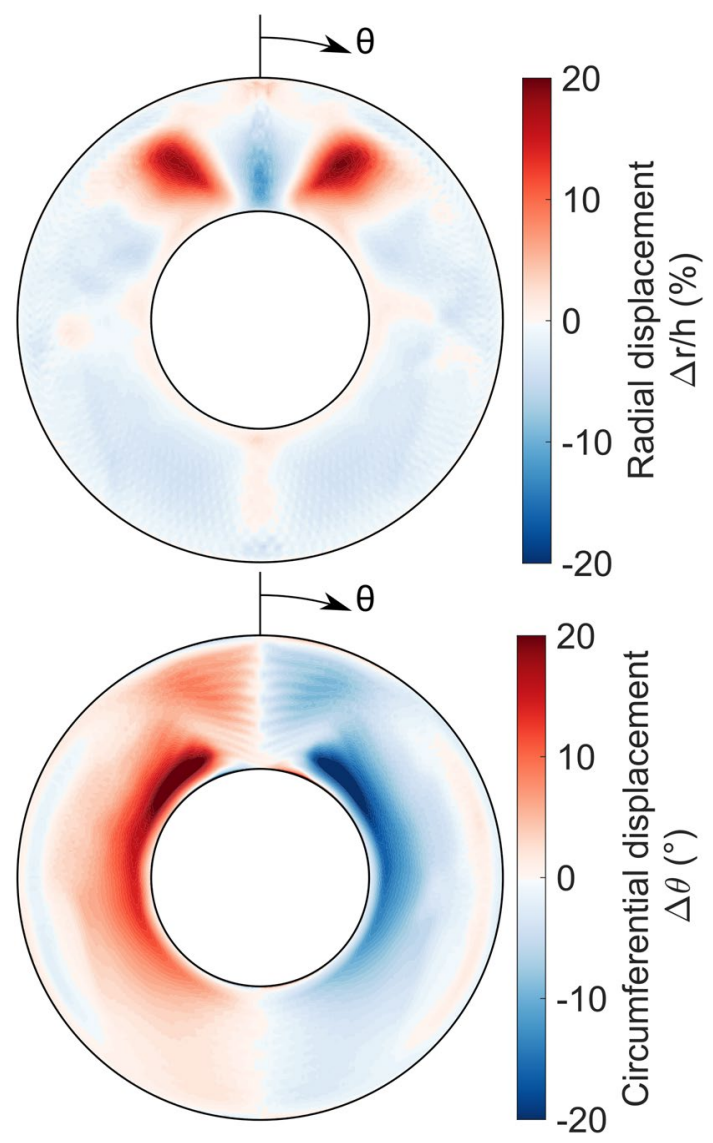

Fig. 10 Radial and circumferential displacements downstream of the gauze for BLI fan at cruise underpredicted. This can be related to the mixing process previously described in Eq. 2. Regardless of the magnitude of the discrepancies, the flow topology has been captured in both the simulations and experiments.

To illustrate the necessity of performing streamline tracking during the iterative design process, the radial and circumferential displacements between the gauze trailing edge and the measurement plane are shown in Fig. 10. The radial displacement has been normalised by the rig span. Overall, small displacements are found across the annulus. However, areas with large gradients in stagnation pressure drop coefficient are associated with larger displacements. Accounting for the displacements with the streamline tracking process allows the solver to modify the gauze geometry at the correct emanating location.

The second flight condition modelled is sideslip, where the aircraft is in level flight with a $4^{\circ}$ sideslip or yaw angle. The sideslip flow component moves from left to right in Fig. 11 (stagnation pressure) and Fig. 12 (swirl), generating a highly distorted asymmetric inflow. Different features can be identified. On the left side, high momentum fluid associated with the acceleration around the intake lip is observed. The interaction of the high momentum flow with the vertical tail plane leads to a strong shockwave boundary layer interaction, which separated the boundary layer at $\theta \approx-15^{\circ}$. A second region of high momentum is observed at the bottom, which is linked to a vortex released from the belly fairing. A large low momentum region is observed at $\theta \approx 45^{\circ}$. This is associated with the larger vertical tail plane wake found with sideslip. In terms of swirl angle, the flow is restricted by the vertical tail plane at the top. Consequently, the flow is forced to migrate anti-clockwise around the fuselage, leading to a strong counter-swirl distortion.

Figure 11 presents the comparison between the target design, numerical predictions and measured stagnation pressure coefficient at the target plane. The methodology can replicate the main flow features of the stagnation pressure
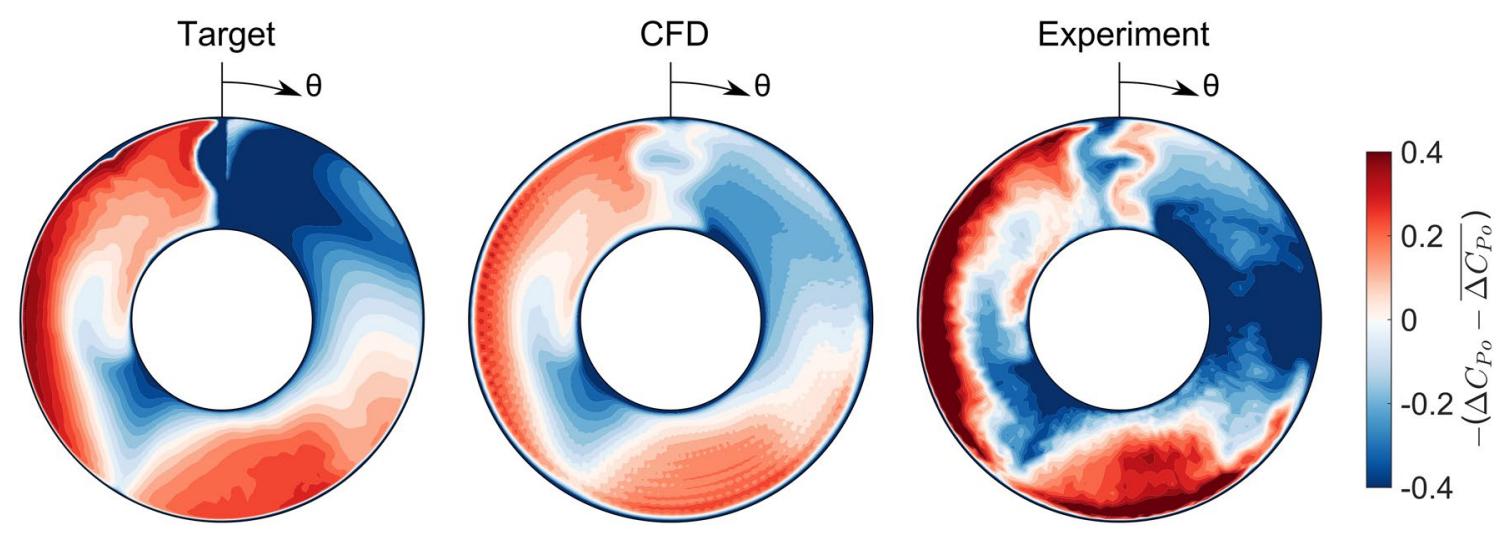

Fig. 11 CFD design and experimental measurements of stagnation pressure coefficient of BLI fan with sideslip condition 

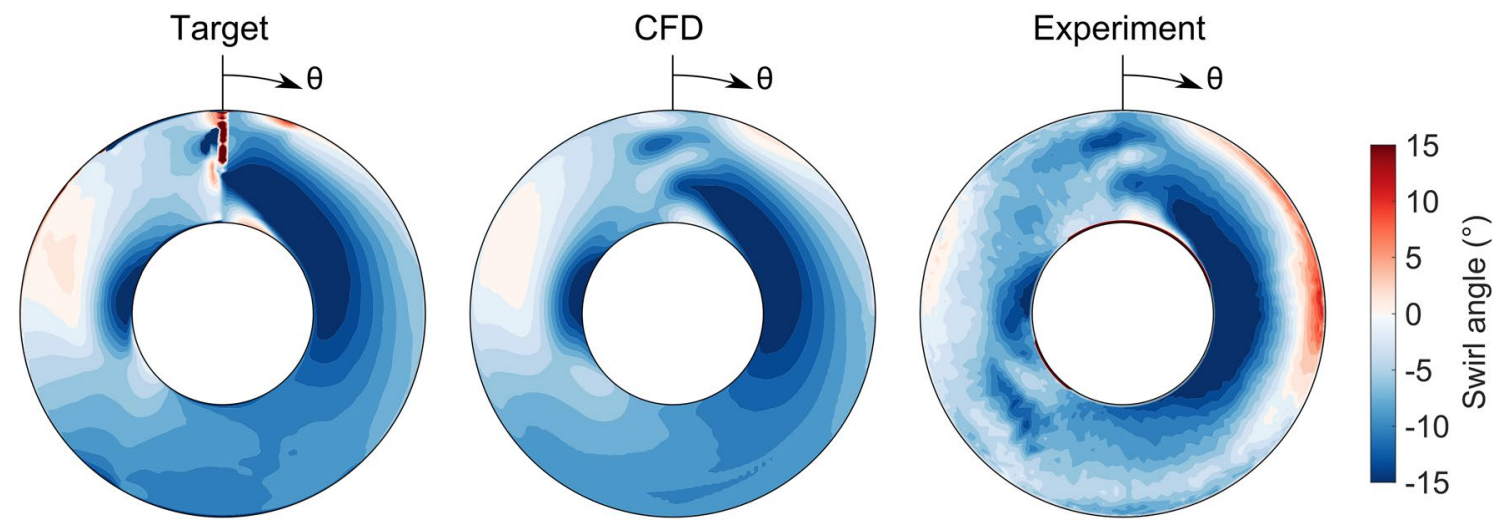

Fig. 12 CFD design and experimental measurements of swirl angle of BLI fan with sideslip condition

distortion. The shape and magnitude of the components are well captured with exception of the large vertical tail plane wake. A shift of almost $90^{\circ}$ in the low momentum region is measured in the rig that is not predicted in the CFD. This could be associated to the underprediction of loss by the CFD solver in the region of interest. Increased loss in the experiment can increase the turning during the mixing process as tangential momentum is conserved. The increased loss could be caused by errors in the modelling in the CFD or imperfections caused by the manufacturing process. A likely candidate for the source of manufacturing error would be roughness within the passages causing increased growth of the gauze passage boundary layers.

Figure 12 presents the swirl angle comparison, where a very good agreement in terms of shape and magnitude is observed. As above, the largest discrepancy has been measured on the right side near the casing. This co-swirling flow is responsible for the circumferential shift in the loss pattern. Despite the small discrepancies, the main features of the flow have been replicated successfully.

\subsection{Compact core compressor}

The proposed gauze technology has been applied to the problem of an axial compressor operating downstream of a short swan neck duct with 12 structural struts passing through it. The duct of interest is shown in Fig. 7 and presented in Taylor et al. (2021). As it has been designed for use in a hybrid air-breathing rocket engine, it has been reduced in length by $30 \%$ compared to current aero engines. In this work, it was found that the structural struts cause significant loss and distortion at the duct outlet plane. In an engine application, this distortion is expected to cause significant problems of loss and stability for the downstream compressor. An optimised design was presented that uses a circumferential splitter blade to help offload the endwalls, which reduces loss and distortion of the duct. The work experimentally measured the 2D flowfield at the duct outlet plane using a stationary suck-down sector test rig for both the datum and the splittered duct design. In this section of the paper, the aim is to design two gauzes to replicate the major flow features of these two experiments so that the downstream effect can be quantified in a rotating compressor rig.

The two target profiles are plotted in Fig. 13. These contour maps show stagnation pressure coefficient, swirl and pitch angle for the datum design on the left and the splittered duct design on the right. In both designs the area of low stagnation pressure at the centre is caused by the effect of the strut. In the datum case, a large hub-side corner separation is convected out towards the casing, while the hub endwall separation can also be seen to roll up mid-way between two struts, shown in the bottom left hand corner of the contour map here. Swirl and pitch angles show the secondary flow that is driven by the loading on the duct endwalls overturning the strut wake. In the splittered duct design, it can be seen that both the depth and extent of low stagnation pressure regions and the angularity of the flow are reduced; therefore, this design is expected to have a reduced detrimental effect on the downstream compressor.

Figure 13 also shows the comparison between the CFD predictions and experimentally measured flowfields at the target plane downstream of the two gauze designs. The depth of the low stagnation pressure regions in the centre of the gauze are underpredicted by the CFD, although the magnitudes between the experiment and the target profiles are comparable. In the datum design this region is concentrated towards the casing of the compressor; this is expected to have a greater effect on the downstream compressor as it is located close to the tip of the rotor blade row. The modelled wake of the strut in the splittered duct is more uniform across the span. Swirl and pitch angles of the flow are also more intense in the experiment, but the main features of the secondary flow are present. Radial flow in the centre of the strut wake is well captured as are the pair of passage 

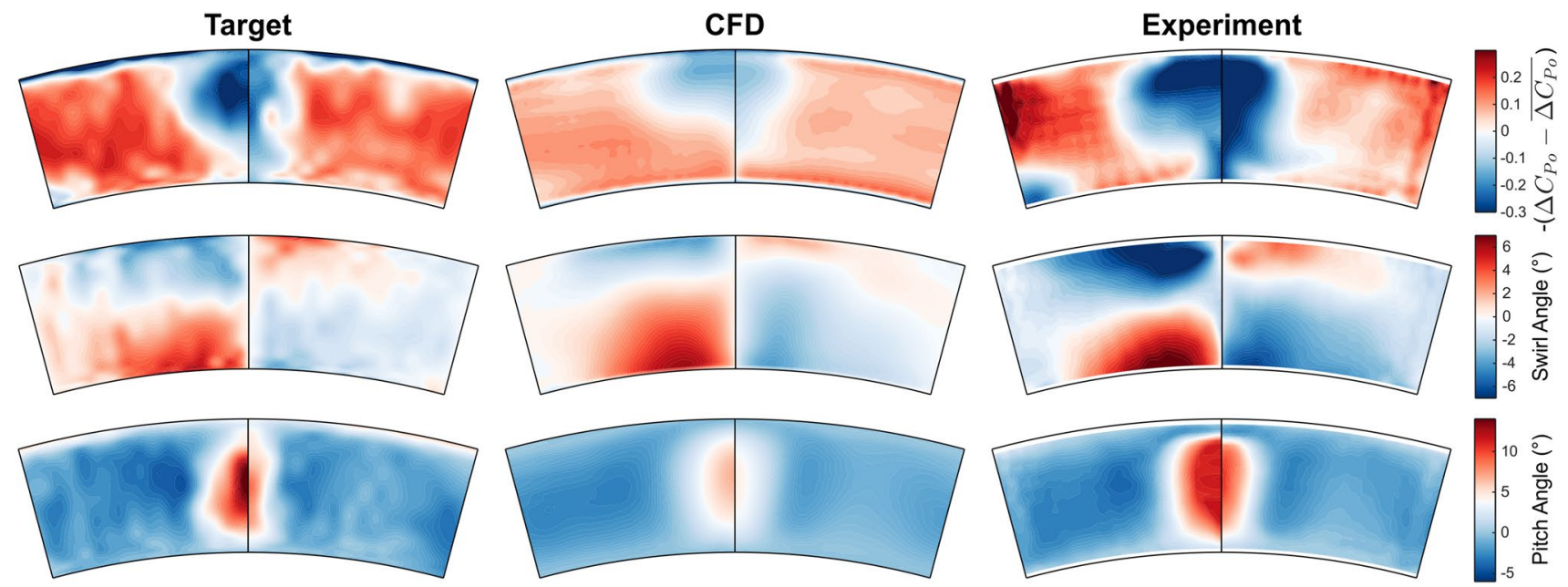

Fig. 13 CFD design and experimental measurements of compact core compressor gauzes. All left contour maps are datum design, all right maps are splittered duct design

vortices that result. The splittered duct has less intense secondary flow and so is expected to have a reduced effect on the operability of the downstream compressor. The wake of the splitter blade itself, present as a ring at $30 \%$ span off the hub is also captured in the experiment, any detrimental effect from this feature can also be captured in the downstream experiment.

Using this methodology, it has been possible to decouple the effects of experimental campaigns on different turbomachinery components and investigate the flow physics more meaningfully. In the published work of Taylor et al. (2021), the loss mechanisms within the duct were investigated. Using the non-axisymmetric complete flow conditioning gauze, the effect of the distortion on a downstream compressor can now be quantified with a very simple and flexible experiment on a rotating rig.

\section{Conclusions}

Complete flow conditioning gauzes can be used to generate any stagnation pressure and swirl or pitch angle pattern that varies in both radial and circumferential directions. By individually tailoring many radial and circumferential blades in a thick additively manufactured gauze, it is possible to model the real distortion conditions seen by turbomachinery components in an engine within a rotating rig or wind tunnel experiment in a laboratory environment. This increases the flexibility of experiments and reduces the space required for infrastructure and their associated costs.

The method is demonstrated for two applications. In the first, a boundary layer ingesting fan mounted at the aft of an aircraft, inflow conditions can be reproduced that model the distortion seen at cruise and at a condition with sideslip. In the second, a compact core compressor, inflow conditions can be reproduced to model the distortion generated by two upstream swan neck ducts, both with structural struts but only one with an optimised splitter blade to offload the diffusion of the endwalls.

The applications given in this paper are only two examples of the type of turbomachinery problems that are going to be encountered in the future. To quantify the effects of flow conditions at the interfaces of tightly integrated engines requires the use of non-axisymmetric complete flow conditioning gauzes. The method outlined in this paper is necessary to improve our understanding of these problems and allows us to achieve the performance needed in the future.

Acknowledgements The authors are grateful for the comments, suggestions, and encouragement of colleagues at the Whittle Laboratory, most notably Tony Dickens, Chris Clark, Sam Grimshaw, Phoenix Tse, Tim Williams, Chez Hall and Rob Miller. The authors would also like to thank Martijn van Sluis at Delft University of Technology for the fan inlet conditions, Fergus Flanagan in collaboration with Reaction Engines for the experimental data, and TURBOSTREAM for the use of their solver.

Author's contributions All authors contributed to the study conception and design. Material preparation, data collection and analysis were performed by all authors. All authors wrote and approved the manuscript.

Funding This research was partially funded by the European Union's Horizon 2020 research and innovation programme under grant agreement no. 723242 .

\section{Declarations}

Conflicts of interest The authors have no conflicts of interest to declare that are relevant to the content of this article. 
Open Access This article is licensed under a Creative Commons Attribution 4.0 International License, which permits use, sharing, adaptation, distribution and reproduction in any medium or format, as long as you give appropriate credit to the original author(s) and the source, provide a link to the Creative Commons licence, and indicate if changes were made. The images or other third party material in this article are included in the article's Creative Commons licence, unless indicated otherwise in a credit line to the material. If material is not included in the article's Creative Commons licence and your intended use is not permitted by statutory regulation or exceeds the permitted use, you will need to obtain permission directly from the copyright holder. To view a copy of this licence, visit http://creativecommons.org/licenses/by/4.0/.

\section{References}

Allen O, Castillo Pardo A, Hall CA (2021) An experimental investigation into the impacts of varying the circumferential extent of tip-low total pressure distortion on fan stability. Proceedings of the ASME Turbo Expo 2021: Turbomachinery Technical Conference and Exposition. Virtual, Online. June 7-11

Arend DJ, Wolter JD, Hirt SM, Provenza A, Gazzaniga JA, Cousins WT, Hardin LW, Sharma O (2017) Experimental evaluation of an embedded boundary layer ingesting propulsor for highly efficient subsonic cruise aircraft. Proceedings of the AIAA 53rd AIAA/ SIAA/SAE/ASEE Joint Propulsion Conference. Atlanta, GA. July 10-12, 2017. AIAA 2017-5041. AIAA. https://doi.org/10.2514/6. 2017-5041

Brandvik T, Pullan G (2010) An accelerated 3D Navier Stokes solver for flows in turbomachines. J Turbomach 133(2):021025. https:// doi.org/10.1115/1.4001192

Bruni G, Taylor J, Krishnababu S, Miller R, Wells R (2020) Squealer tip treatment design for axial compressors. Proceedings of the ASME Turbo Expo 2020: Turbomachinery Technical Conference and Exposition. Volume 2A: Turbomachinery. Virtual, Online. 2020. V02AT32A035. ASME. https://doi.org/10.1115/ GT2020-14906

Castillo Pardo A, Hall CA (2021) Aerodynamics of boundary layer ingesting fuselage fans. J Turbomach 143(4):041007. https://doi. org/10.1115/1.4049918

Frohnapfel DJ, Lowe KT, O'Brien WF (2020) Development, analysis, and validation of a simultaneous inlet total pressure and swirl distortion generator. Proceedings of the ASME Turbo Expo 2020: Turbomachinery Technical Conference and Exposition. Volume 2A: Turbomachinery. Virtual, Online. 2020. V001T01A038. ASME. https://doi.org/10.1115/GT2020-16125

Guimarães T, Lowe KT, O'Brien WF (2018) StreamVane turbofan inlet swirl distortion generator: mean flow and turbulence structure. J Propuls Power 34(2):340-353. https://doi.org/10.2514/1.B36422

Gunn EJ, Hall, CA (2014) Aerodynamics of boundary layer ingesting fans. Proceedings of the ASME Turbo Expo 2014: Turbine
Technical Conference and Exposition. Vol. 1A. Düsseldorf, Germany, June 16-20, 2014. V01AT01A024. ASME. https://doi.org/ $10.1115 / G T 2014-26142$

Harjes L, Bode C, Grubert J, Frantzheld P, Koch P, Friedrichs J (2020) Investigation of jet engine intake distortions caused by crosswind conditions. J Glob Power Propuls Soc 4:48-62. https://doi.org/ 10.33737/jgpps/118875

Houghton T, Day I (2011) Stability enhancement by casing grooves: the importance of stall inception mechanism and solidity. J Turbomach 134(2):021003. https://doi.org/10.1115/1.4002986

Lucas JR, O'Brien,WF, Ferrar AM (2014) Effect of BLI-type inlet distortion on turbofan engine performance. Proceedings of the ASME Turbo Expo 2014: Turbine Technical Conference and Exposition. Volume 1A. Düsseldorf, Germany, 2014. V01AT01A037. ASME. https://doi.org/10.1115/GT2014-26666

Place JMM, Howard MA, Cumpsty NA (1996) Simulating the multistage environment for single-stage compressor experiments. J Turbomach 118(4):706-716. https://doi.org/10.1115/1.2840926

Roach PE (1987) The generation of nearly isotropic turbulence by means of grids. Int J Heat Fluid Flow 8(2):82-92. https://doi.org/ 10.1016/0142-727X(87)90001-4

Seitz A, Habermann AL, Peter F, Troeltsch F, Castillo Pardo A, Della Corte B, van Sluis M, Goraj Z, Kowalski M, Zhao X, Grönstedt T, Bijewitz J, Wortmann G (2021) Proof of concept study for fuselage boundary layer ingesting propulsion. Aerosp 8(1):16. https:// doi.org/10.3390/aerospace8010016

Taylor JV (2019) Complete flow conditioning gauzes. Exp Fluids 60(3):35. https://doi.org/10.1007/s00348-019-2682-9

Taylor JV, Miller RJ (2016) Competing three-dimensional mechanisms in compressor flows. J Turbomach 139(2):021009. https://doi.org/ $10.1115 / 1.4034685$

Taylor JV, Conduit B, Dickens A, Hall C, Hillel M, Miller RJ (2020) Predicting the operability of damaged compressors using machine learning. J Turbomach 142(5):051010. https://doi.org/10.1115/1. 4046658

Taylor JV, Flanagan F, Dunlop A, Grimshaw SD, Miller RJ (2021) Super aggressive s-ducts for air breathing rocket engines. J Turbomach 143(6):061015. https://doi.org/10.1115/1.4050596

van Sluis M (2020) D3.03 - Final PFC aircraft aerodynamic design and performance. Delft. CENTRELINE Project Public Deliverable Report

Wheeler APS, Miller RJ, Hodson HP (2006) The effect of wake induced structures on compressor boundary-layers. J Turbomach 129(4):705-712. https://doi.org/10.1115/1.2720499

Publisher's Note Springer Nature remains neutral with regard to jurisdictional claims in published maps and institutional affiliations. 